\title{
Incommensurate dynamics of resonant breathers in Josephson junction ladders
}

\author{
M. V. Fistul, A. E. Miroshnichenko and S. Flach \\ Max-Planck-Institut für Physik komplexer Systeme, Nöthnitzer Strasse 38, D-01187 Dresden, Germany \\ M. Schuster and A. V. Ustinov \\ Physikalisches Institut III, Universität Erlangen-Nürnberg, D-91058 Erlangen, Germany
}

(November 15, 2018)

\begin{abstract}
We present theoretical and experimental studies of resonant localized resistive states in a Josephson junction ladder. These complex breather states are obtained by tuning the breather frequency into the upper band of linear electromagnetic oscillations of the ladder. Their prominent feature is the appearance of resonant steps in the current-voltage $(I-V)$ characteristics. We have found the resonant breather-like states displaying incommensurate dynamics. Numerical simulations show that these incommensurate resonant breathers persist for very low values of damping. Qualitatively similar incommensurate breather states are observed in experiments performed with Nb-based Josephson ladders. We explain the appearance of these states with the help of resonance-induced hysteresis features in the $I-V$ dependence.

$74.50+\mathrm{r}, 05.45 \mathrm{Yv}, 63.20 \mathrm{Ls}$
\end{abstract}

A lot of attention has been recently devoted to the theoretical and experimental study f $_{\text {intrinsic dynamic }}$ localized states in various physicalle, chemical and biological systems 3 . These peculiar inhomogeneous states, called discrete breathers, appear in spatially homogeneous (familtonian or dissipative) nonlinear discrete systems [- It was found that these states manifest themselves in the spectra of molecules and solids, and can determine the energy transfer in complex biological systemsd. Discrete breathers have been observed e.g. in weakly coupled optical waveguides, in antiferromagnetigsplids and in systems of interacting Josephson junctions 813 .

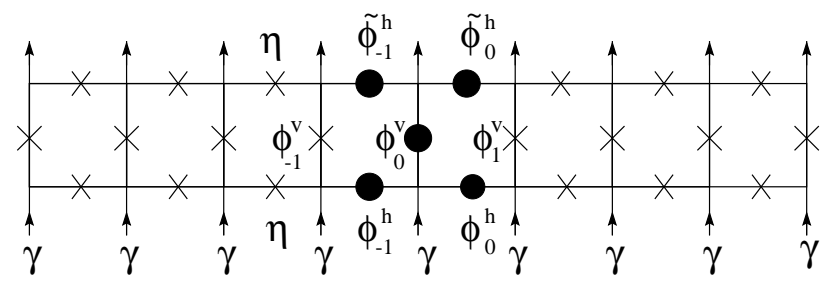

FIG. 1. Josephson junction ladder. Crosses mark the individual junctions. Arrows indicate the direction of external current flow (dc bias $\gamma$ ). The black circles indicate the junctions that are in the whirling (resistive) state. The breather state displaying up-down symmetry is shown,

Especially in the latter example, namely homogeneously dc driven Josephson junction ladders (JJLs) (see Fig. 1), an enormous diversity of breather states has been observed. The JJL consists of small Josephson junctions of two types, "horizontal" and "vertical" ones, which are, respectively arranged perpendicular and parallel to the applied dc bias current $\gamma$. Breather states in a JJL are characterized by spatially localized voltage patterns as a few Josephson junctions are in the resistive (whirling) states (the black circles in Fig. 1) while the rest of the JJL junctions are in the superconducting state. The dynamics of breather states crucially depends on two parameters: the anisotropy $\eta=\frac{I_{c H}}{I_{c V}}$, where $I_{c H}$ and $I_{c V}$ are respectively the critical currents of horizontal and vertical junctions, and the discreteness parameter (normalized inductance of the cell), $\beta_{L}$.

The complete dynamics of JJLs is determined by the time dependent Josephson phases $\phi_{i}^{v}(t)$ (vertical), $\phi_{i}^{h}(t)$ (lower horizontal), and $\tilde{\phi}_{i}^{h}(t)$ (upper horizontal), and is governed by the Kirchhoff's laws and the flux quantization law. By making use of the resistively shunted model for Josephson imnctions the following set of equations has been obtained 17 :

$$
\begin{aligned}
& \ddot{\phi}_{n}^{v}+\alpha \dot{\phi}_{n}^{v}+\sin \phi_{n}^{v}=\gamma+\frac{1}{\beta_{L}}\left(\triangle \phi_{n}^{v}+\nabla \phi_{n-1}^{h}-\nabla \tilde{\phi}_{n-1}^{h}\right) \\
& \ddot{\phi}_{n}^{h}+\alpha \dot{\phi}_{n}^{h}+\sin \phi_{n}^{h}=-\frac{1}{\eta \beta_{L}}\left(\nabla \phi_{n}^{v}+\phi_{n}^{h}-\tilde{\phi}_{n}^{h}\right) \\
& \ddot{\tilde{\phi}}_{n}^{h}+\alpha \dot{\tilde{\phi}}_{n}^{h}+\sin \tilde{\phi}_{n}^{h}=\frac{1}{\eta \beta_{L}}\left(\nabla \phi_{n}^{v}+\phi_{n}^{h}-\tilde{\phi}_{n}^{h}\right),
\end{aligned}
$$

where we use the notations $\triangle f_{n} \equiv f_{n-1}-2 f_{n}+f_{n+1}$ and $\nabla f_{n} \equiv f_{n+1}-f_{n}$. Here, the unit of time is the inverse of the plasma frequency $\omega_{p}$, and $\alpha$ is the damping constant for a single junction. The dc voltage drop across a single junction is defined through a time average $\langle\dot{\phi}(t)>$. Of crucial importance for the rich variety of breather states is the presence of a nonzero dissipation in JJLs. This dissipation ensures that breathers can survive resonant interaction with extended linear electromagnetic waves (EWs), while their dynamic complexity may actually increase at the same time. Indeed, these complex resonant breather states have been predicted in [Ref. 8], experimentally observed in [Ref. 15], and analyzed in detail in [Ref. 14]. The condition of the appearance of such a res- 
onant breather state is the matching of the breather frequency $\Omega$ or its higher harmonics with the frequency $\omega(q)$ of one of the cavity modes of the ladder, $\omega(q)=m \Omega$. The breather frequency $\Omega$ is determined by the smallest nonzero dc voltage drop in the JJL, and the spectrum of cavity modes $\omega(q)$ has been derived previously using the linearization of the Eqs. (11) around the superconducting ground state14 16 :

$$
\begin{aligned}
\omega_{ \pm}^{2} & =F \pm \sqrt{F^{2}-G}, \\
F & =\frac{1}{2}+\frac{1}{\beta_{L} \eta}+\frac{1}{2} \sqrt{1-\gamma^{2}}+\frac{1}{\beta_{L}}(1-\cos q), \\
G & =\left(1+\frac{2}{\beta_{L} \eta}\right) \sqrt{1-\gamma^{2}}+\frac{2}{\beta_{L}}(1-\cos q) .
\end{aligned}
$$

For a finite size ladder with open boundary conditions, the spectrum of linear waves is discrete and characterized by the following choice of allowed wave number values:

$$
q_{l}=\frac{l \pi}{N+1} \quad l=0,1,2 \ldots, N,
$$

where $N$ is the number of cells. Note here, that the resonant breather state is still a strictly periodic solution in time. Its resonant character is revealed by large amplitude librations of the Josephson phases in the whole JJL. Resonant breather states are manifested by resonant steps in the current-voltage $(I-V)$ characteristics, i.e. by a region where the voltage saturates at a fixed value while the bias $\gamma$ is increased.

The presence of the resonant breather state leads to the appearance of hysteresis in the $I-V$ curve. Consequently there exist particular ranges of $\gamma$ where both resonant and non-resonant breathers can be stable. However, these two types of breathers are characterized by different (incommensurate) frequencies $\Omega$, and moreover, the frequencies depend in a different manner on $\gamma$. Thus, the question naturally appears: Is it possible for a breather state to be both resonant and nonresonant at the same time in a JJL? This is important because nondissipative systems support only time-periodic breathers, while dissipative systems typically need an external time-dependent drive to generate quasiperiodic dynamics. The idea here is that the spatial nonuniformity of the states (localized breathers) acts in a similar way to external time-dependent drives, so that even a dc bias is enough to obtain quasiperiodic (in time) states.

Here we will consider a region of parameters where the breather strongly interacts with the EWs. We find that for low values of damping $\alpha$ a novel breather-like state with incommensurate (aperiodic) dynamics persists. This incommensurate resonant state can be viewed as the mixture of resonant and non-resonant breathers in the JJL. Moreover, we also report on the experimental observation of this peculiar breather state in an extremely underdamped JJL. The incommensurate resonant breather-like state leads to two successive voltage jumps in the $I-V$ curve.
To study the breather dynamics we perform direct numerical simulations of the set of equations (i1). To establish a large dc current region where a strong resonant interaction of the breather state with the EWs occurs we choose the discreteness parameter $\beta_{L}=0.378$. The ladder consists of $N=10$ cells and the anisotropy parameter $\eta=0.49$. We impose open boundary conditions and use 4th order Runge-Kutta method for integration. The initial value of the dc bias was $\gamma=0.8$. We select proper initial conditions that lead to the relaxation of the system into a particular breather state of up-down symmetry with one resistive vertical jumction, as shown in Fig. 1. Similar to our previous work 4 , after a waiting time of 500 time units we use the next 500 time units to calculate the time averaged characteristics of the state. Then the dc bias $\gamma$ was decreased (increased) by a step of $\Delta \gamma=0.001$ and the procedure was repeated. We checked that our results do not change upon further increase of the waiting time. The $I-V$ characteristics, that is the dependence of the averaged voltage drop across the resistive Josephson junction on the dc bias, was monitored. The simulations were carried out for two different values of damping $\alpha=0.1$ (see Fig. 2) and $\alpha=0.025$ (see Fig. 3).

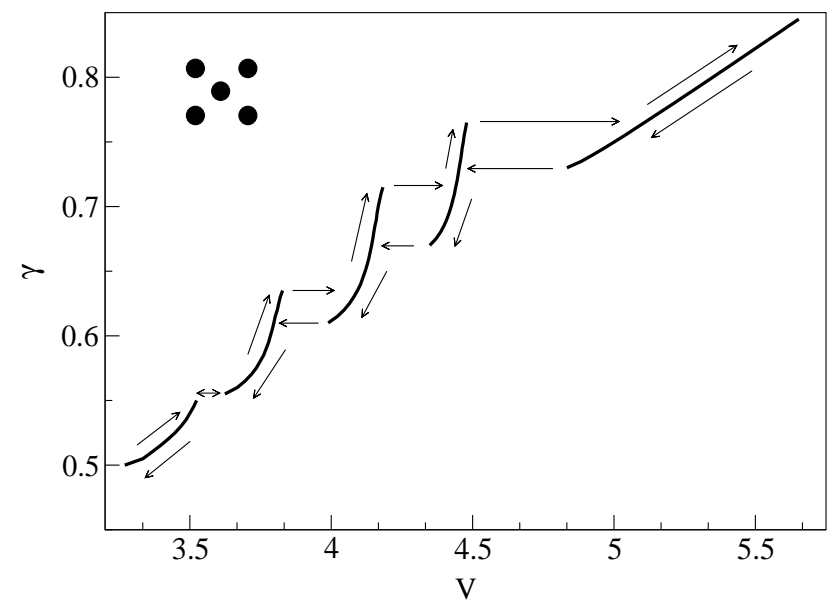

FIG. 2. $I-V$ characteristics for $\alpha=0.1, \beta_{L}=0.378$, $\eta=0.49$. Arrows show the dc bias current increase (decrease). The dc voltage pattern of the breather state is presented by the black circles.

In the case of a moderate value of damping $\alpha=0.1$ we obtain a non-resonant breather state for dc bias current values down to $\gamma \geq 0.72$. This breather state displays a complete up-down symmetry, $\phi_{i}^{h}(t)=-\tilde{\phi}_{i}^{h}(t)$. The $I$ - $V$ curve is linear in this regime. In the region of moderate dc current bias values resonant steps appear in the $I$ - $V$ curve (Fig. 2). The presence of these steps indicates a strong resonant interaction between the breather state and EWs as the condition $\omega_{+}\left(q_{l}\right)=2 \Omega$ is satisfied. The observed steps correspond to resonances with 
cavity modes with particular values of $l=2,4,6,8$. As the JJL is biased at the steps, a resonant type of the breather state is realized. This state conserves the structure of the dc voltage pattern but is characterized by a large ac voltage component of the superconducting Josephson junctions. Independent of the observed dynamical complexity, all breather states (resonant or nonresonant type) found for this value of damping $\alpha$ display a time-periodic behavior.

However, the situation changes drastically as we lower the dissipation down to $\alpha=0.025$. First, the breather state strongly interacts with EWs if the condition $\omega\left(q_{l}\right)=\Omega$ is matched. Secondly, we observe only a single resonant step corresponding to the particular cavity mode $(l=2)$ excitation. Under these conditions we found that the switching from the non-resonant breather state 18 appearing for large values of dc voltages, to the resonant breather occurs in the form of two successive voltage jumps (see, Fig. 3a).

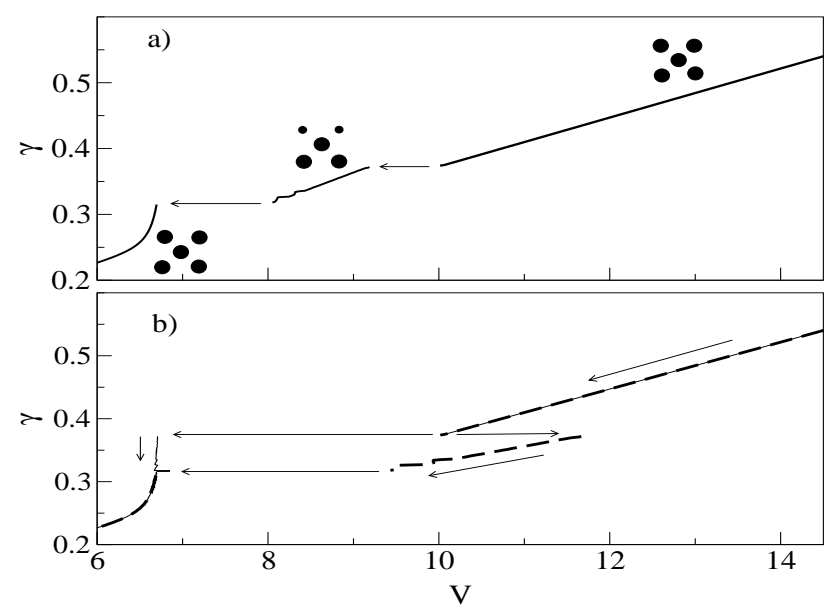

FIG. 3. $I-V$ characteristics for $\alpha=0.025, \beta_{L}=0.378$, $\eta=0.49$. Arrows indicate the voltage jumps and show the decrease of the dc bias current. a) Result for voltage drop across the vertical junction. The size of the black circles indicates the relative values of dc voltage drops. b) Result for twice the voltage drop across the upper (solid line) and lower (dashed line) horizontal junctions.

Thus, in the particular region of the dc bias current $(0.31<\gamma<0.38)$ the up-down symmetry of the dc voltage pattern is violated and a novel resonant breatherlike state with incommensurate (aperiodic) dynamics appears. Indeed, as the system jumps to this state there are at least two incommensurate frequencies, namely $\Omega_{1} \simeq 3.4$ and $\Omega_{2} \simeq 5.3$. Moreover, with decreasing dc bias the value of $\Omega_{1}$, practically does not change, in contrast to $\Omega_{2}$. A closer look at the $I-V$ curves of the horizontal junctions (Fig. 3b) also shows that the upper (lower) horizontal junctions display the resonant (non-resonant) behavior. Thus we can conclude that this novel incommensurate breather-like state can be viewed as a coexistence of resonant and non-resonant junction behavior in the JJL. We note here that similar aperiodic breather states were found in a wide range of parameters $\beta_{L} \simeq 1$ and $\eta \leq 1$

By making use of a dc analysis 8.14 , i.e. neglecting ac components of the Josephson current, we find that the voltage jumps $\Delta V_{h}$ and $\Delta \tilde{V}_{h}$ of the non-resonant and resonant horizontal junctions have to satisfy the relationship:

$$
\Delta V_{h}=\frac{\Delta \tilde{V}_{h}}{1+2 \eta}
$$

Moreover, the presence of the incommensurate resonant breather state is characterized by a lower resistance $\propto$ $(\alpha(2 \eta+1))^{-1}$ as compared to the non-resonant case. Indeed, our simulations show that the dc voltage jump of the lower horizontal junctions is approximately two times smaller than the corresponding dc voltage jump of the upper horizontal junctions, in full accordance with the dc analysis.

In order to characterize an incommensurate breather state more precisely we calculate the Fourier transform of the time dependent voltage $\dot{\phi}_{1}^{v}(t)$ of the vertical junction (see Fig. 4), which is in the superconducting state and the closest one to the core of the breather.

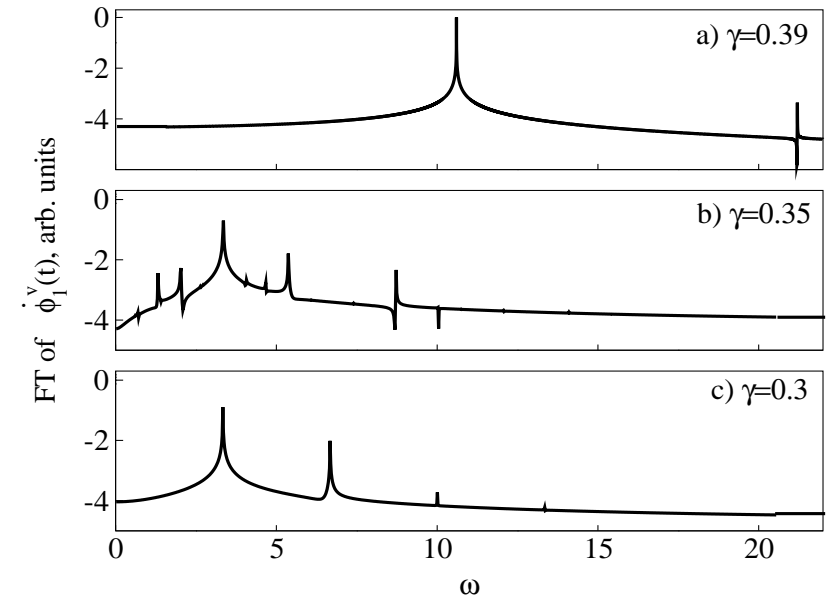

FIG. 4. The Fourier transform (FT) of the ac voltage $\dot{\phi}_{1}^{v}(t)$. Three different values of dc bias current are shown and the logarithmic scale for FT was used.

If the breather state is the periodic one, i. e. for large (Fig. 4a) and small (Fig. 4c) values of dc bias current, the Fourier transform contains the breather frequency $\Omega$ and higher harmonics. However, in the intermediate region of the dc bias as the breather displays incommensurate dynamics, the Fourier transform becomes more rich (Fig. 4b). We observe two different harmonics $\Omega_{1}$ and $\Omega_{2}$, and the various combination frequencies $m \Omega_{1}+n \Omega_{2}$, where $n$ and $m$ are integers. 
The incommensurate breather-like states analyzed above were initially observed in experiments which we performed using Nb-based Josephson junction ladders. The experimental layout is similar to the one presented in [Ref. 9] and corresponds to the schematic shown in Fig. 1. The measurements were performed in liquid helium at a temperature of $T=4.2 \mathrm{~K}$. The data we present were taken from a 10 cell open-ended Josephson ladder of anisotropy $\eta=0.49$. By chosing a relatively low critical current density of $j_{c} \approx 100 \mathrm{~A} / \mathrm{cm}^{2}$ for the samples we achieved a discreteness parameter of $\beta_{L}=0.62$, which makes it possible for a breather to be strongly resonant with EWs. At the same time, the low damping value of $\alpha=0.025$ was sustained.

The breather state was created artificially by initially applying a local bias current $\gamma_{l}$ to one of the vertical junctions, and thus, forcing the switching of this junction into the resistive state. After that we decreased $\gamma_{l}$ and simultaneously increased the homogeneous bias current $\gamma$. This procedure, described in detail in [Ref. 9], finally yields the desired breather state in the presence of an uniformly supplied bias current. In this paper we focused on an up-down symmetric breather state with dc voltage pattern shown in Fig. 1. The $I-V$ curves were obtained by sweeping the bias current $\gamma$ and measuring the respective voltage drops across the junctions. For convenience, we plot normalized voltage $v=V / V_{0}$ with $V_{0}=\frac{\Phi_{0}}{2 \pi} \omega_{p}=$ $84.3 \mu \mathrm{V}$, and normalized bias current $\gamma=I_{\text {ext }} / I_{c V}$ with $I_{c}=20.5 \mu \mathrm{A}$.

For the sample \#1 the breather state was created at a dc bias current $\gamma=0.38$. The acquired $I-V$ curve, as shown in Fig. 5, displays a linear, non-resonant behavior until $\gamma=0.35$ (see Fig. 5). The breather state is symmetric, and the absolute values of the horizontal junction voltages $\left|V_{h}\right|$ and $\left|\tilde{V}_{h}\right|$ are just one half of the vertical junction voltage, $\left|V_{v}\right|$. As the dc bias is decreased further, the switching to the resonant breather state takes place. This switching occurs through an intermediate state (see Fig. 5a). Indeed, in this region of $0.32<\gamma<0.35$ we observe the symmetry-broken state with $\left|\tilde{V}_{h}\right| \neq\left|V_{h}\right|$. Moreover, the ratio of voltages $\left|\tilde{V}_{h}\right| /\left|V_{h}\right|$ continuously changes as $\gamma$ decreases (see Fig. 5b). For the values of $\gamma<0.32$ the symmetric breather state recovers but is now a resonant breather state.

For the sample \#2 we observe a similar intermediate state appearing in the process of the switching from the non-resonant to the resonant breather state (see Fig. 6). In this experiment carried out in the presence of a small magnetic field (the normalized magnetic flux per cell was less than 0.1 ), the $I-V$ curve of the non-resonant (lower) horizontal junction (see Fig. 6b) displays a voltage jump similar to the one found in the numerical studies (see Fig. 3). The ratio of the voltages and the corresponding rotation frequencies of the horizontal (upper and lower) junctions increases as $\gamma$ decreases. The measured $I-V$ curves in both cases qualitatively look similar to the current-voltage characteristics obtained by numerical simulations of (1). Thus, the predicted breather state displaying aperiodic dynamics is clearly observed (compare Figs. 3 and 5,6).
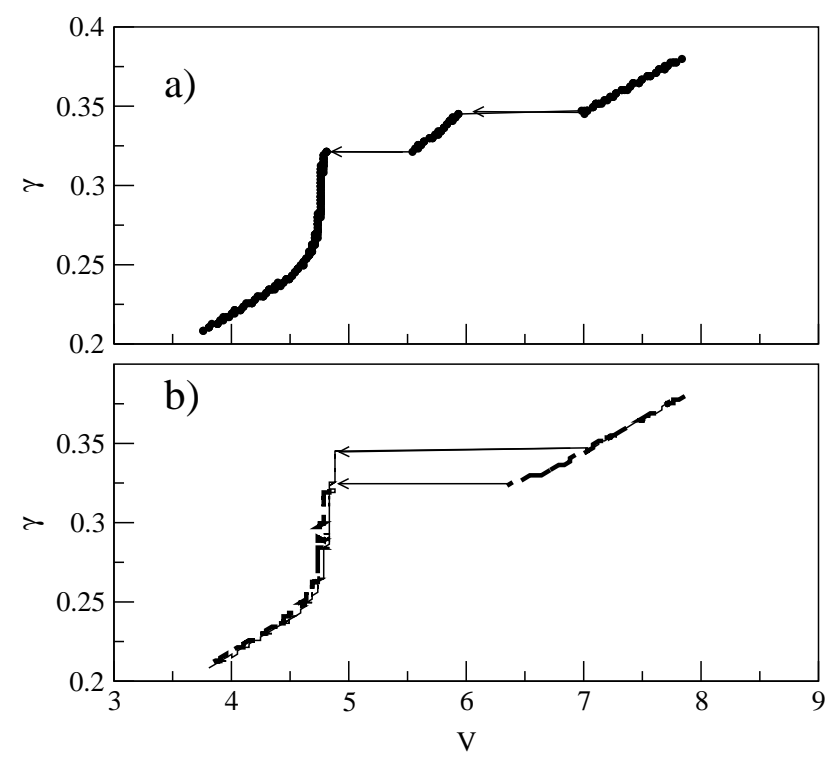

FIG. 5. Measured $I-V$ curves (sample \#1) of an initially symmetric breather state created at a dc bias of $\gamma=0.38$ : (a) voltage drop across vertical junction, (b) voltage drops across upper (thin solid line) and lower (dashed line) left horizontal junctions. The breather state with incommensurate dynamics is formed for $0.32<\gamma<0.35$. The fine structure superimposed on the curve is due to reduced experimental resolution in these measurements.

However, there are several differences between the experimental observations and the numerical analysis of (1). Firstly, in contrast to numerics we did not observe the voltage jump of the non-resonant horizontal junction in the measurement shown in Fig. 5, and secondly, in Fig. 6 we observe just a tiny voltage difference between upper and lower horizontal junctions in the bias region above the incommensurate state. This particular behavior is not yet explained. We want to stress that the incommensurate breather state was observed under various experimental conditions, which were partly influenced by magnetic fields in the vicinity of the studied sample.

In conclusion, we found an inhomogeneous resistive state showing aperiodic dynamical behavior by making use of a numerical analysis of (1). Similar incommensurate breather states were observed experimentally in niobium-based underdamped Josephson junction ladders. The incommensurate breather states are characterized by a resonant behavior of some horizontal junctions and a non-resonant behavior of the other horizontal junctions. Such states appear in the process of a switching from symmetric non-resonant to symmetric resonant breather state. The origin of these particular breather states is a strong resonant excitation of EWs by the breather state leading to the resonance-induced hysteresis in the $I-V$ curve. Thus, we argue that the aperiodic inhomogeneous 
dynamic states can present a generic feature of dissipative (but extremely underdamped) lattices.

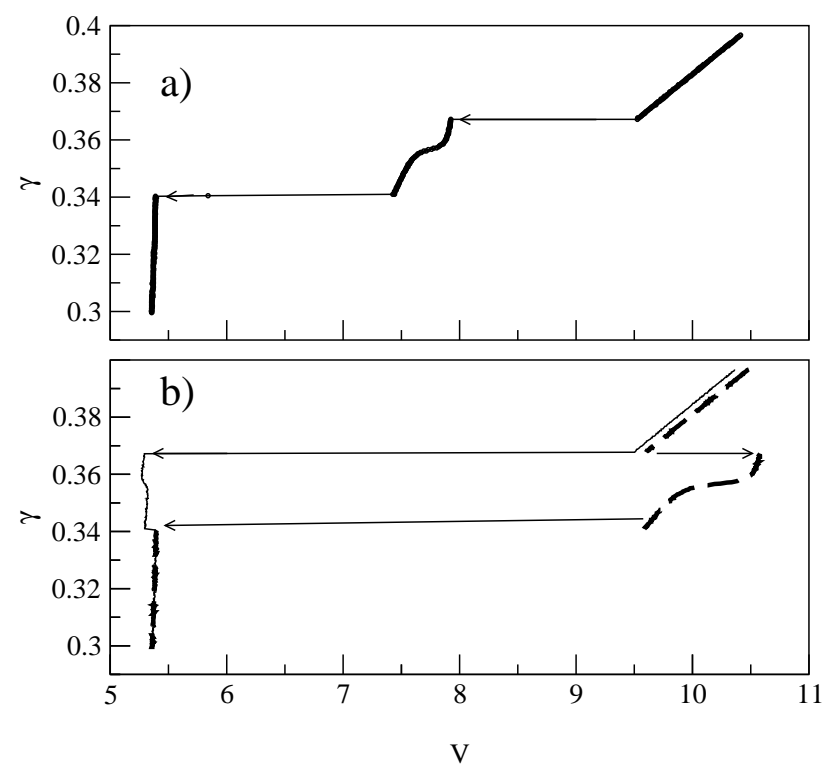

FIG. 6. Measured $I-V$ curves (sample \#2) of an initially symmetric breather state created at a dc bias of $\gamma=0.4$ : (a) voltage drop across vertical junction, (b) voltage drops across upper (thin solid line) and lower (dashed line) left horizontal junctions. The breather state with incommensurate voltages is formed for $0.34<\gamma<0.37$.

We thank A. Benabdallah for useful discussion. This work was supported by the Deutsche Forschungsgemeinschaft and by the European Union under the RTN project LOCNET HPRN-CT-1999-00163.

${ }^{1}$ B.I. Swanson, J.A. Brozik, S.P. Love, G.F. Strouse, A.P. Shreve, A.R. Bishop, W. -Z. Wang, and M.I. Salkola, Phys. Rev. Lett., 82, 3288 (1999).

${ }^{2}$ U.T. Schwarz, L. Q. English, and A.J. Sievers, Phys. Rev. Lett., 83, 223 (1999)

${ }^{3}$ S. Aubry and G. Kopidakis, Cond. Mat./0102162, (2001).

${ }^{4}$ M. Peyrard, Europhys.Lett., 44, 271 (1998).

${ }^{5}$ S. Aubry, Physica D, 103, 201 (1997).

${ }^{6}$ S. Flach and C. R. Willis, Physics Report, 295, 181 (1998).

${ }^{7}$ S. Aubry, G. Kopidakis, A. M. Morgante, G. P. Tsironis, to be published in Physica B.

${ }^{8}$ E. Trias, J. J. Mazo, and T. P. Orlando, Phys. Rev. Lett, 84, 741 (2000).

${ }^{9}$ P. Binder, D. Abraimov, A. V. Ustinov, S. Flach, and Y. Zolotaryuk, Phys. Rev. Lett, 84, 745 (2000).

${ }^{10}$ P. Binder, D. Abraimov, and A. V. Ustinov, Phys. Rev. E, 622858 (2000).

${ }^{11}$ L. M. Floría, J. L. Marin, P. J. Martinez, F. Falo, and S. Aubry, Europhys. Lett., 36, 539 (1996)
12 S. Flach and M. Spicci, J. Phys.: Condens. Matter, 11, 321 (1999).

13 E. Trias, J. J. Mazo, A. Brinkman, and T. P. Orlando, Physica D 156, 98 (2001).

14 A. E. Miroshnichenko, S. Flach, M. V. Fistul, Y. Zolotaryuk, and J. B. Page, Phys. Rev. E, 64, 066601 (2001).

15 M. Schuster, P. Binder, and A. V. Ustinov, to be published in Phys. Rev. E (2002).

${ }^{16}$ P. Caputo, M. V. Fistul, A. V. Ustinov, B. A. Malomed, and S. Flach, Phys. Rev. B, 59, 14050 (1999).

${ }^{17}$ G. Grimaldi, G. Filatrella, S. Pace, and U. Gambardella, Phys. Lett. A, 223, 463 (1996)

${ }^{18}$ In the case of a small dissipation we also found that a nonresonant breather state displays a broken dynamic symmetry. Thus, the breather state with a complete up-down symmetry becomes unstable and the stable (non-resonant) states are characterized by a relationship: $\left\langle\phi_{0}^{h}(t)+\tilde{\phi}_{0}^{h}(t)\right\rangle=$ $\pm \pi$. The properties of these peculiar breather states will be reported elsewhere. 